\title{
Dependence of ocean wave return levels on water depth and sampling length: a focus on the South Yellow Sea
}

Article

Accepted Version

Creative Commons: Attribution-Noncommercial-No Derivative Works 4.0

Feng, X., Li, H., Feng, X., Zhao, J. and Feng, W. (2021) Dependence of ocean wave return levels on water depth and sampling length: a focus on the South Yellow Sea. Ocean Engineering, 219. 108295. ISSN 0029-8018 doi: https://doi.org/10.1016/j.oceaneng.2020.108295 Available at https://centaur.reading.ac.uk/93750/

It is advisable to refer to the publisher's version if you intend to cite from the work. See Guidance on citing.

To link to this article DOI: http://dx.doi.org/10.1016/j.oceaneng.2020.108295

Publisher: Elsevier

All outputs in CentAUR are protected by Intellectual Property Rights law, including copyright law. Copyright and IPR is retained by the creators or other copyright holders. Terms and conditions for use of this material are defined in the End User Agreement.

www.reading.ac.uk/centaur 
Central Archive at the University of Reading

Reading's research outputs online 
Dependence of ocean wave return levels on submarine topography and sampling length: a focus on the South Yellow Sea

\author{
Xi Feng ${ }^{1,2 *}$, Huichao Li $^{1}$, Xiangbo Feng ${ }^{3}$, Jiajing Zhao ${ }^{1}$, Weibing Feng' \\ ${ }^{1}$ Key Laboratory of Coastal Disaster and Defence (Hohai University), Ministry of \\ Education, Nanjing, Jiangsu Province, China \\ ${ }^{2}$ College of Harbor, Coastal and Offshore Engineering, Hohai University, Nanjing, \\ Jiangsu Province, China. \\ ${ }^{3}$ National Centre for Atmospheric Science and Department of Meteorology, University \\ of Reading, UK \\ *Corresponding author: Xi Feng, Doctorate, E-mail: xifeng@hhu.edu.cn
}

\begin{abstract}
A deterministic Extreme Value Analysis method is of particular importance in an engineering-oriented context. In this paper, three extreme value analysis (EVA) methods, in which annual maxima (Type I), monthly maxima (Type II) or the peaksover-threshold (Type III) are fitted into Generalized Extreme Value distribution (GEV) function or Generalized Pareto distribution (GP) function, are used to estimate return values of significant wave height. Sensitivity of return levels of significant wave height to water depth and sampling length is vigorously investigated, based on the 40 years of high-resolution wave hindcasts for the South Yellow Sea (SYS). We find that for 100year return level $\left(H_{100}\right)$, amongst the three methods, Type I method mostly provides the largest estimates in the shallow waters and are most sensitive to water depth, whilst estimates from Type II are the smallest and least sensitive to water depth. Additionally, the GP model shows more confidence for long-term return level estimates (e.g. $H_{100}$ ) and the least vulnerable to the sampling length, but produces wider confidence level than the GEV model for short-term level estimates. In the SYS, the return level estimates are significantly reduced with a longer sample length. However, we find that the reduction is closely related to the long-term trend in extreme wave heights, rather than due to the sampling effect. From deep to shallow waters, spatial inhomogeneity of return levels increases, which should be considered in the engineering practice.
\end{abstract}

Keywords: Extreme Value Analysis; Generalized Extreme Value distribution; Generalized Pareto distribution; Peak-over-threshold; Return level, Sampling length.

\title{
1. Introduction
}

A wide range of engineering applications requires the accurate information on extreme waves, partially related to the coastal and offshore area protections. Examples include the determination of design parameters for breakwaters, seawalls and offshore platforms. Meanwhile, extreme waves can also cause coastal erosion and threaten the 
safety of fisheries and the coastal marine ecosystems. Extreme value analysis (EVA) is used to estimate the return values or occurrence probabilities of extreme waves. In the EVA procedure, tail values of observed or simulated wave metrics are fitted to a theoretical function, from which the return values or occurrence probabilities of extreme waves at certain levels are calculated (Goda, 1992; Muir and El-Shaarawi, 1986; Muraleedharan et al., 2012). Among the EVA estimates, the Generalized Extreme Value (GEV) distribution model is popularly used due to its flexibility and good-fitness in simulating extreme waves under the various conditions (e.g. Niroomandi et al., 2018; Menéndez et al., 2009). The GEV model can be specified with the Gumbel (type I of GEV) and Weilbulls (type III of GEV) (e.g. Shi et al., 2019; Li et al., 2018; Mathiesen et al., 1994). In addition to the GEV model, a Generalized Pareto (GP) distribution model has also been used in the wave return level estimations (e.g. Coles, 2001; Méndez et al., 2006; Hawkes et al., 2008; Thompson et al., 2009), which is also known as the Peaks-Over-Threshold (POT) due to the sampling method.

Choosing the EVA approach for extreme wave height estimation varies on a case-by-case basis. Before the analysis for wave return levels at a location, there is normally very little information indicating the best or most-proper methods, likely due to the very localized features of extreme waves. Goda et al. (1993) conducted an intercomparison between Weibull and Fisher-Tippett distributions (type I and type II), and found that the return level estimates are dependent on the sampling variability. This work is mostly derived from the statistics perspective, while the effects of geophysical variations such as water depth were not considered. In contrast, van Vledd et al. (1993) compared the wave height 100-year return levels with the POT and annual maxima values used in the type I and type III methods of GEV distribution, and found that there are small differences between the methods. Note that van Vledd et al. (1993) used wave measurement data at only location in the Norwegian coast, and the comparison was made for two different periods (9-years vs. 20-years). Mazas and Hamm (2011) revisited the dataset in van Vledd et al. (1993) together with another single-site wave records for the Gibraltar Strait, and suggested two difference EVA methods for these two different sites regarding the fitness of observed extreme wave heights to the statistical models. Mazas and Hamm (2011) also found that the wave return levels in the Gibraltar Strait is less sensitive to the statistical methods than in the Norwegian coast. Contrastingly, in the Chesapeake Bay, Niroomandi et al. (2018) found that the statistical methods can considerably alter the wave return levels. Therefore, the performance of EVA methods varies with regions and the length of data. The suggestions for the most suitable methods in one place may not be valid in another place. Ideally, for each marginal sea or coastal region, a vigorous and systematic evaluation on the feasibility of EVA methods in estimating the wave height return levels should be performed, although there has always been lack of it. In this paper, we aim to systematically evaluate the different EVA methods for return level estimations in the SYS, including uncertainties of return levels related to by intrinsic factors (e.g. location and data source) and epistemic factors (e.g. statistical models and sampling length).

The temporal variability and trends of extreme marine events are expected to have an essential effect on the estimated return levels (e.g. Ross et al., 2017; Wahl and 
Chambers, 2015; Feng and Tsimplis, 2014; Grinsted et al., 2014; Arns et al., 2013; Méndes et al. 2006; 2008; Menéndez et al., 2008; Wang and Swail, 2001, 2002). An increase or positive anomalies of the size/occurrence of extreme events over a period would imply the larger values of return level. This could contaminate the effect of the data sampling length on return levels, in which the samples are assumed to be independent of time (i.e. at a normal distribution). Extreme wave heights have been seen to increase or decrease by $* * \mathrm{~cm} / \mathrm{yr}$ in the western North Pacific over the last decades (REF), likely related to $* *$ what reasons**. It remains unclear whether such significant changes in the open ocean will be able to translate into the marginal seas such as the SYS. The bathymetric refraction and shallow water in the SYS may damp the amplitudes of trend and variations in extreme waves from the open sea, especially for the swells, causing these trends and variability of waves in the SYS to be undetectable. In this paper, we will investigate how the long-term variations of waves in the SYS affect the sensitivity of wave return levels to the data sampling length.

The SYS is located adjacent to one of the most developing coastal regions of China, with an enormous submarine deltaic system, known as the Radial Sand Ridges (JRSR). Over the last decades, the bathymetry and coastlines of the SYS become more complex due to intensive human activities, including land-reclamation and harbor development (Feng et al., 2019), which could potentially cause distinct changes in the local-wave generations and remote-wave propagations. However, long-term hydrological observations are very sparse, adding uncertainty to the return level estimations for marine extremes (Feng et al., 2010, Yang et al., 2014, Feng and Tsimplis, 2014, Feng et al., 2018). There have been limited studies sophistically evaluate the applicability of the EVA methods to this region, despite the importance from the engineering-oriented perspective.

The objectives of this paper include: 1) to identify a most suitable EVA method for the SYS, as a function of water depth and data sampling length, for the engineeringoriented purposes; 2) to evaluate the uncertainties in return level estimations due to the intrinsic factors (water depth) and epistemic factors (sampling length and EVA methods); and 3) to detect whether the long-term trend in extreme wave climate can affect the uncertainties of return level to the above factors. The paper is structured as follows. Section 2 describes the EVA methods and the wave datasets of the SYS. Section 3 gives the detailed analysis on the return values of extreme wave height, and the sensitivities to water depth and data sampling length. Section 4 discusses the impact of long-term variations of wave height on the return level sensitivity analysis, including the comparisons to previous studies and implications for engineering practice. We provide our conclusions in Section 5.

\section{Methodology}

\subsection{Numerical dataset}

A forty-year long numerical hindcasted dataset spans from 1979-2018 with focus on the SYS $\left(119^{\circ} \mathrm{E}-124^{\circ} \mathrm{E}\right.$ and $31^{\circ} \mathrm{N}-35^{\circ} \mathrm{N}$ ) (Figure 1) was used as the sampling dataset in this study. The SWAN wave model, a third-generation wave model, 
developed at Delft University of Technology, that computes random, short-crested wind-generated waves in coastal regions and inland waters (Boojj,1999) (v41.20), with unstructured module was used to construct the wave fields. The unstructured computational grid contained 20097 triangular elements and 10429 nodes, with resolution gradually varying from $1.5 \mathrm{~km}$ to $10.8 \mathrm{~km}$ in the coastal areas with an increase of $10-15 \%$. The directions from $0-360^{\circ}$ was divided into 36 bins, and the frequencies of the simulated waves are divided into 32 frequencies from $0.04 \mathrm{~Hz}$ to $1 \mathrm{~Hz}$ evenly. The time step of the simulation was set to 60s. The northern, southern and eastern boundaries were set as open boundaries. The offshore swells were implemented and forced into the study domain via the northern, eastern and southern open boundaries. Physics considered in the model included white capping, wave breaking and bottom friction.

The contour colors in Figure $1 \mathrm{~b}$ represent the bathymetry, which was obtained from nautical chart sourced of year 2010. The shallow areas off the coastlines were dominated by the Jiangsu Radial Sand Ridges system (JRSR). The effects of Yangtze River flow and sea level variations were not considered in the wave hindcast, i.e. assuming water depth was constant during the simulation period. The submarine sediment movement and the geomorphologic evolution over the 40 years were limited in the JRSR. Since the main focus of this study is on the extreme wave caused by atmospheric factors, the coastline and bathymetry of the SYS were assumed unchanged to avoid uncertainties introduced by human inventions or morphological changes.

The model was driven by the ERA-Interim reanalysis winds at $10 \mathrm{~m}$-height above sea surface, obtained from ECMWF (https://www.ecmwf.int/) and the offshore swell-forcing were extracted from the operational ocean wave predictions of NOAA/National Weather Service based on WaveWatchIII (WWIII) (https://polar.ncep.noaa.gov/waves/). In this study, we used 40 years (1979-2018) of ERA-Interim data at 6 hourly interval, with a spatial resolution of approximately $80 \mathrm{~km}$. NCEP reanalysis winds were also used for comparing waves forced with ERA-Interim, during the period of 2009-2014 when measurements were available. The model was parameterized with reference to model configurations on the China adjacent seas from previous studies (Shi et al., 2019; Liang et al., 2014; Li et al., 2016).Wave hindcasts were verified with in-situ wave measurements: Buoy $1\left(120.81^{\circ} \mathrm{E}, 33.29^{\circ} \mathrm{N}\right)$, Buoy 2 $\left(121.57^{\circ} \mathrm{E}, 32.15^{\circ} \mathrm{N}\right)$, and Buoy $3\left(121.98^{\circ} \mathrm{E}, 32.26^{\circ} \mathrm{N}\right)$ ( Figure 2).

The SWH at all three stations were better been hindcasted by ERA-Intrim from ECMWF than CFSR from NCEP regarding larger correlation coefficient (R) and higher Willmott Skill Score (defined in Supplemental Information). Willmott Skill Score equals to 1 meaning a perfect hindcast. In our case, the Willmott Skill Score was all greater than 0.7 (Table 1). The biases between the modeling results and observations were very limited to $-0.09 \mathrm{~m}$ at Buoy $1,0.08 \mathrm{~m}$ at Buoy 2, and $0.03 \mathrm{~m}$ at Bouy 3, which implied that the model can well capture the wave climate over a long time. The three buoys were all deployed at the apex of the RSR. The water depth is $25.5 \mathrm{~m}$ (based on Mean-Sea-Level) at Buoy 1,10m at Bouy 2 and 20. $9 \mathrm{~m}$ at Buoy 3. The offshore waves were either been buffered on ebb shoals or sheltered by the emerged ridges, which 
means the SWH at the three buoys was very sensitive to the local bathymetry. Thus, the mean SWH at Buoy 1 and Buoy 3 exceeded no more than $0.5 \mathrm{~m}$ and at Buoy 2 was even smaller. Additionally, due to the resolution of wind dataset, the wind field close to the shore could also introduce biases. Considering the factors that can cause the deviations, the performance of our model can be considered as feasible, regarding such a long-time simulation. Moreover, the modeling accuracy was comparable to and even better than the previous numerical studies (e.g. Shi et al., 2019; Li et al., 2018; Liang et al., 2014). As the calibration of the model was not the focus of this study, the model validation are not expanded here. More details about the model validation are provided in the Supplemental Information (refer to S1).

\subsection{Sampling methods}

The SWH was used to quantify the extreme wave climate in the study area. For instance, the $1 \%$ annual maximum wave height, so-called the 100 -year return value of SWH $\left(H_{100}\right)$, refers to the SWH values being exceeded at $1 \%$ chance at any given year. The extreme return level has been widely used as designing wave parameter in coastal and ocean engineering. In this study, three samplings methods were used to estimate the extreme return values.

A traditional but convenient approach for estimating $H_{100}$ depends on the annual maximum wave heights, which is widely recommended officially (e.g. Oceanic Administration of China, Federal Emergency Management Agency of United States). The annual maximum wave height can be attributed to many factors, such as a storm wave event, a rogue wave, or a passage of an abrupt atmospheric disturbance such as meteo-tsunamis (Niu and Chen, 2019). However, such an extreme circumstances could appear more than once per year. Besides, for samples with short-period, annual maxima might fail to supply enough data for accurate estimates. Two supplementary sampling methods were used to make up for the deficiency and increase feasible sampling-size: 1) picking extreme values with monthly-maxima and the return value of SWH can be transformed into $H_{T}=H_{T^{\prime}}$, where $T^{\prime}=T / 12 ; 2$ ) peak-over-threshold (POT) method.

The POT method is more flexible and can reflect the occurrence of extreme weather more accurately (Niroomandi et al. 2018; Menendez et al. 2008). Values that exceed a certain threshold from a continuous measurement were counted into peak values to make up for the sparseness in the tail values resulted from the annual maximum method. To implement the POT sampling method, the SYS shelf was divided into 20 blocks for thresholds' determination. Each block covered an area with latitude and longitude $1^{\circ} \times 1^{\circ}$, except for the JRSR where a finer block size of $0.5^{\circ} \times 0.5^{\circ}$ was applied. The resolution of the blocks depended on the variability in geographic and bathymetric patterns shown in Figure 1b. The naming of each block is as following: letter D represents the deep-water area, P denotes the periphery of the JRSR, and the north, center, and south of JRSR are represented by N, R and S, respectively.

In practice of the POT method, two critical parameters, which are the threshold of SWH where peak waves ought to exceed, and the time interval between two consecutive but independent extreme wave events, need to be taken carefully. A proper 
selection of the threshold and the time interval ought to ensure that the occurrences of extreme weathers were taken into account as much as possible. Whilst too low a threshold would result in underestimation in return level and too short a time interval would cause duplicate numbers within the same extreme event. The minimum time interval to assure independence between consecutive storms was chosen to be 3 days (e.g. Menéndez et al. 2006). Meanwhile, the previous studies have consistently demonstrated that 3-day as the optimal value for time span as little variability in POT was observed with varying time span (e.g. Niroomandi et al., 2018; Méndez et al., 2006, 2008). Following the pioneering works, a 3-day time span has been picked and applied over the SYS.

This study used a combination of the mean excess function (MEF) plot and the mean residual life (MRL) plot to make the selection of the threshold to implement POT sampling method. The MEF was proposed by scholar McNeil in 1997. It can be defined as follow:

$$
e_{n}(\mu)=E(X-U \mid x>\mu)=\frac{\sum_{i=1}^{n}\left(X_{i}-\mu\right)}{n}
$$

where $\mu$ represents the threshold, and $x_{i}(i=1,2, \cdots, n)$ represents the sample observation exceeding the threshold. In the MEF plot, a set of points with $\left\{\mu, e_{n}(\mu), \mu>0\right\}$ were plotted with $\mu$ set as the horizontal axis and $e_{n}(\mu)$ set as the vertical axis. The threshold selection was determined when a linear platform (without abrupt ups and downs) appeared after $\mu$ exceeding a specific value. The upper panel of Figure 3 shows the MEF plots for 3 representative blocks. On the other hand, Coles (2001) proposed that an optimal threshold should also satisfy that the shape parameters of the fitted GP distribution be in a stable linear state. In the stable interval, a threshold as large as possible should be used to achieve the original intention of selecting extreme values. Thus, the MRL plot has been put forward, in which $\mu$ remained as the horizontal axis, while the vertical axis was replaced by the shape parameter in the GP model. The threshold depending on the MRL plot was achieved when the trend of the scatter plot became linear. The low panel of Figure 3 shows the MRL plot of the three representative blocks with a $95 \%$ confidence interval for the shape parameter estimates between red lines.

Given a time series of SWH, the optimal threshold was determined from an overlapped range of $\mu$, where both the MEF plot and the MRL plot became stable and linear. Then a specific value of $\mu$ was determined by finding the location where the gradient of the corresponding curves both tended to zero. In the example shown in Figure 3, the $\mu$ of the three representative regions (D2, P4, R3) were: $2.3 \mathrm{~m}, 2.5 \mathrm{~m}$, and $2.1 \mathrm{~m}$, respectively. The thresholds in all the 20 blocks are shown in Table 2 . The 40year averaged sampling dataset for each sampling method was mapped in Figure 4.

\subsection{Statistical models}

Following previous studies (e.g. Niroomandi et al., 2018; Méndez et al., 2006, 2008), GEV model and GP model were both used by this study for $H_{100}$ estimation. First, the annual maxima and monthly maxima of the 40 -year long dataset was fitted 
into the GEV distribution function for $H_{100}$ assessment. The cumulative distribution function of GEV model follows:

$$
G(z, \mu, \sigma, \xi)_{G E V}=\left\{\begin{array}{l}
\exp \left[-\left(1+\xi \frac{z-\mu}{\sigma}\right)^{-1 / \xi}\right], \xi \neq 0 \\
\exp \left[-\exp \left(-\frac{z-\mu}{\sigma}\right)\right], \quad \xi=0
\end{array},\right.
$$

where $z\left(z_{1}, z_{2}, z_{3}, \cdots, z_{n}\right)$ represents the independent random variable subject to GEV distribution and here is extreme wave height; $\mu$ is the location parameter, $-\infty<\mu<$ $+\infty$; $\sigma$ is the scale parameter, with $\sigma>0$; and $\xi$ is the shape parameter, $-\infty<\xi<$ $+\infty ; 1+\xi \frac{z-\mu}{\sigma}$ needs to be positive. The shape parameter $\xi$ determines three types of GEV models, with Gumbel distribution subject to $\xi=0$, Frechet distribution for $\xi<0$, Weibull distribution for $\xi>0$. The parameters $\mu, \sigma, \xi$ were determined by the maximum likelihood estimates (MLE) at each grid point. Given a return period (T) (e.g. $T=100$ year) and a location, the return value of SWH can be obtained by the following equation:

$$
H_{T_{G E V}}=\left\{\begin{array}{lr}
\mu-\frac{\sigma}{\xi}\left\{1-\left[-\ln \left(1-\frac{1}{T}\right)^{-\xi}\right]\right\}, \xi \neq 0 \\
\mu-\sigma \ln \left[-\ln \left(1-\frac{1}{T}\right)\right], & \xi=0
\end{array} .\right.
$$

The graphic analysis based on the cumulative distribution function (CDF) plots and the quantile-quantile (Q-Q) plots was used to judge the fitness of the distribution function. The fitness of GEV distribution are shown in Figure 5 and Figure 6 . It can be seen from the CDF plots that the fitted values (blue line) and the theoretical values (red line) are consistent with the distribution trend, which proves that the GEV distribution is suitable for the two sampling methods. In the Q-Q plots, the observational points (blue markers) are also conforming to the theoretical distribution (red dashed line). With sampling source based on the annual-maxima (Figure 6), a slight deviation from the theoretical distribution can be observed, which is typical in EVA because of the uncertainty aroused from the sparseness of samples.

The GP model performs well in fitting the exceeding values over a threshold. Thus we fit the POTs into the GP model, and the GP model has the following cumulative distribution function:

$$
\mathrm{G}(z, \sigma, \xi)_{G P}=\left\{\begin{array}{ll}
1-\left(1+\xi \frac{z}{\sigma}\right)^{-1 / \xi}, \xi \neq 0 \\
1-\exp \left(-\frac{z}{\sigma}\right), \quad \xi=0
\end{array},\right.
$$

where $z\left(z_{1}, z_{2}, z_{3}, \cdots, z_{n}\right)$ represents the independent random variable (here is extreme wave height) subject to the GPD distribution, $\sigma$ and $\xi$ are the scale and shape parameters, respectively. The parameter $\sigma$ and $\xi$ were determined using MLE. The solution of the return value of SWH $\left(H_{T}\right)$ corresponding to the return period $T$ is as follows: 


$$
H_{T_{G P}}=\left\{\begin{array}{ll}
\mu+\frac{\sigma}{\xi}\left(T^{\xi}-1\right), & \xi \neq 0 \\
\mu+\sigma \ln (T), & \xi=0
\end{array} .\right.
$$

To evaluate the performance of GP model in fitting the POT samples, the distribution function of excess proposed by McNeil (1997) was applied:

$$
F_{u}(x-u)=\operatorname{Pr}\{X-u \leq x \mid X>u\}=\frac{F(x)-F(u)}{1-F(u)}, x \geq 0,
$$

where $u$ is the threshold, $X\left(x_{1}, x_{2}, x_{3}, \cdots, x_{n}\right)$ represents the independent random variable subject to the distribution $F, F(x)<1$. McNeil (1997) suggested that, for a sufficiently high thresholds $u$, the distribution function of the excesses $\left(F_{u}(x-u)\right)$ will be approximated by GP distribution. The performance of GP model in fitting the tail values are shown in Figure 7. In the figure, the blue dots shows the empirical distribution by Equ.(6) and the black line indicates the GP distribution. Dotted line in red represents the boundaries of the GP distribution with a deviation in $u$ of $\pm 5 \%$. It was found that the GP with the thresholds selected (Table 2) gave the best performance to the empirical distributions. Table 3 summarized the types of methods for assessing the return level of extreme wave heights.

\section{Results}

3.1 Maps of $H_{100}$ from three types of sampling method

Maps of $H_{100}$ vary to each other by using different methods (Figure 4). In the offshore regimes, $H_{100}$ determined by Type II method is lower than that calculated by Type I or Type III method by approximately 20-30\%. Type III provides the largest $H_{100}$ in the southeast of the domain, while Type I gives the highest $H_{100}$ in the northeast. Spatial nonuniformity of $H_{100}$ using Type I is larger than those from other two methods. Exception happens in the JRSR, where large waves are much reduced $(<2 \mathrm{~m})$. The $H_{100}$ estimates are generally below $1 \mathrm{~m}$ over the sand ridges, reflecting significant energy dissipation due to shallow water effects.

Standard deviation (STD) of $H_{100}$ due to varied statistical methods at each grid point was calculated (Figure 5a). In nearshore and coastal areas (water depth $<30 \mathrm{~m}$ ), the difference between varied methods is limited with STD $<0.2 \mathrm{~m}$. In the offshore area (e.g.P1, P2, D3, D5), the difference is greater with $S T D>0.5 \mathrm{~m}$. The method is regarded more conservative if outputs larger value of $H_{100}$ and is determined at each grid point and shown in Figure 5b. The GPD method with POT samples is more conservative for most of the coastal areas and the east offshore areas. The GEV method with annual maximum values whereas gives more conservative estimates in the north and south.

\subsection{Effects of water depth and sampling length on $H_{100}$}

The above sub-sections analyzed the spatial variations of $H_{100}$ based on 40 years of SWH in the SYS. In the following two sub-sections, we will continuously discuss the effects of sampling length and water depth on the wave-return-heights (e.g. $H_{100}$ ). At each grid point, the sensitivity of wave-return-heights to sampling length is 
quantified by using Equ.5:

$$
\alpha=\frac{H_{100, N}-H_{100,40}}{H_{100,40}} \times 100 \%
$$

where $\alpha$ is a non-dimensional coefficient, subscript $N$ represents the sampling length in units of year. Moreover, we classified $H_{100, N}$ into four regimes depending on the water depth in range of $0-10 \mathrm{~m}, 10-20 \mathrm{~m}, 20-40 \mathrm{~m}$ and $>40 \mathrm{~m}$, which represent intertidal area, submarine deltaic area, spherical of JRSR, and offshore area, respectively.

Figure 6 shows the tendency of spatially-averaged $\alpha$ for each regime with changing $N$. The largest variation in $H_{100}$ happens to Type I method $(|\alpha|$ maximizes around 15\%). The Type II and Type III method whereas are not less sensitive to $N$, with $|\alpha|$ within $5 \%$. This is because for Type I method, sampling source were mostly generated by extreme weathers, thus that longer sampling length is required for obtaining stable $H_{100}$.

In regimes from inter-tidal area to the spherical of JRSR $(0-40 \mathrm{~m}), H_{100}$ would be overestimated from $H_{100,40}$ with shorter $N$. However, the overestimation tends to reduce as water depth gets shallower. The inter-tidal regime $(0-10 \mathrm{~m})$ sees the least variability with sampling length. In the offshore regime (water depth>40m), $H_{100, N}$ tends to be underestimated with decreasing $N$.

\subsection{Effects of water depth and sampling length on different return levels}

\subsubsection{Uncertainty in estimating $\boldsymbol{H}_{T, \boldsymbol{N}}$}

We then evaluated the best-estimation and confidence interval (CI) of return value of SWH $H_{T, N}$ in different methods, against different return periods (T; e.g. 10-year, 20-year, 50-year).The 95\% CI for a given variable $\left(\widehat{x}_{i}\right)$ with significant level $\alpha$ follows the formula:

$$
\hat{x}_{C I}=\hat{x}_{i} \pm u_{1-\frac{\alpha}{2}} \hat{S}_{T}
$$

where $\widehat{x}_{C I}$ represents the upper or lower boundary of $\widehat{x}_{i}$; by setting $\alpha=0.1, u_{1-\frac{\alpha}{2}}=1.96$, $\hat{S}_{T}$ is the arithmetic square root of the approximate variance of $\hat{x}_{i}$, which was determined via MLE:

$$
\begin{gathered}
\hat{s}_{T}^{2}=\operatorname{var}\left(\hat{x}_{i}\right)=\left(\frac{\partial x_{i}}{\partial \alpha}\right)^{2} \operatorname{var}(\alpha)+\left(\frac{\partial x_{i}}{\partial \xi}\right)^{2} \operatorname{var}(\xi)+\left(\frac{\partial x_{i}}{\partial u}\right)^{2} \operatorname{var}(u)+2 \frac{\partial x_{i}}{\partial \alpha} \frac{\partial x_{i}}{\partial \xi} \operatorname{cov}(\alpha, \xi)+ \\
2 \frac{\partial x_{i}}{\partial \alpha} \frac{\partial x_{i}}{\partial u} \operatorname{cov}(\alpha, u)+2 \frac{\partial x_{i}}{\partial \xi} \frac{\partial x_{i}}{\partial u} \operatorname{cov}(\xi, u)
\end{gathered}
$$

where $\mu, \sigma$ and $\xi$ are parameters in GEV and GPD functions, refer to Eq. (1-4), var and $\mathrm{cov}$ are operators for calculating self-variance and covariance between pairs of parameters and $\hat{x}_{i}$ is $H_{T, N}$. In Figure 7, each column provides the regime-averaged estimation of $H_{T, N} \mathrm{~s}$ and regional-averaged CIs, from each type of method. In each plot, the shaded area stands for $\mathrm{CI}$, with narrower areas implying more confident estimations of $H_{T, N}$. 
Both the best-estimations of $H_{T, N}$ and the associated CIs reveal distinct dependence on water depth. For intertidal regimes $H_{10}, H_{20}$, and $H_{50}$ are all within the range of $1-2 \mathrm{~m}$ on average, while they increase to $\sim 3 \mathrm{~m}$ for seas with median water depth (20-40m) and over $4 \mathrm{~m}$ for offshore regime (>40m). The CIs of $H_{T, N}$ in the offshore regime are 3-4 times those in the nearshore regime.

Figure 7 also shows that CIs of $H_{T, N}$ are the largest using Type III method, and the smallest using Type II method. As return period $T$ increases, CI of $H_{T, N}$ increases when using Type I or Type II, whereas CI decreases when using Type III method. Nevertheless, as sampling length $N$ increases, $\mathrm{CI}$ in all cases converges, meaning more reliable estimates of $H_{T, N}$.

\subsubsection{Spatial dispersion of estimated $\boldsymbol{H}_{T, N}$}

We further used the standard deviation (STD) of the $H_{T, N} \mathrm{~S}$ at all grid points for a given regime to quantify the spatial variability of return levels. The STD was calculated by Eq. (8):

$$
S T D=\left(\frac{1}{n-1} \sum_{i-1}^{n}\left(\hat{x}_{i}-\bar{x}\right)^{2}\right)^{1 / 2}
$$

where $x_{i}$ represents the return value of SWH $H_{T, N}$ at $i_{t h}$ grid point for a given $T$ and a given sampling-length $N$, and $\bar{x}$ represents $H_{T, N}$ averaged over a given regime.

In Figure 8 wider shaded area suggests greater spatial nonuniformity within one regime. There are no apparent distinctions between STDs of different return levels. The spatial variability is not clearly sensitive to different types of method either. Although spatial variability is slightly larger when using Type I, for small $N(<10$-year) and large $T$ (>50-year). For each case, STD is $\sim 1.0 \mathrm{~m}$ where water depth is within $40 \mathrm{~m}$, and it reduces up to $50 \%$ in waters with depth $>40 \mathrm{~m}$.

\section{Discussion}

\subsection{Implications of uncertainties of return level estimation}

Uncertainties in return level of SWH are generated from both intrinsic factors (e.g. spatial variability due to effects of submarine topography) and epistemic factors (e.g. inevitable errors introduced by the models, sparseness of sampling data). The above results show that epistemic uncertainty dominant over intrinsic uncertainty in return level estimates for most of the open ocean. Moreover, uncertainties due to the statistic models expands as sampling length shortens.

Explicitly, when estimating return value of SWH, the GEV model has more confidence in estimating return levels for small $T$ ( $<50$ years), while the GPD model is more reliable in estimating return levels for large $T$ ( $\geq 50$ years). For seas off the intertidal regime, when sampling length $N$ is less than 20 years, annual-maxima based sampling method has more uncertainties in estimating $H_{50}, H_{100}$, which can be substituted by monthly-maxima or POT based sampling methods. While for estimating shorter return levels, e.g. $H_{10}$ and $H_{20}$, the POT sampling-method has more uncertainties. For intertidal and nearshore regions (water depth $<10 \mathrm{~m}$ ), as long as 
sampling length is larger than 10 years, all methods have similar uncertainties in estimating various return levels.

The spatial variability is relatively small in open seas but large in the nearshore regime. This implies for practice, such as buoy-based estimation of return value of SWH, spatial variability cannot be ignored in nearshore areas. Precise or dense placement of buoy measurement, along with other auxiliary measures such as numerical simulation is required. Distinct regional differences may introduce uncertainty in return level estimations based on just single- or sparse-matrix of buoy deployment.

\subsection{Comparison to other studies}

The mapping of the return levels of extreme SWH from this study were carefully compared to the previous extreme wave studies (e.g. Shi et al., 2019; Li et al., 2018; Li et al., 2016; Chen et al., 2013 and etc.) and proved to very close to the previous ones in magnitude. For example, the estimates of $H_{100}$ were $2.4 \mathrm{~m}, 2.1 \mathrm{~m}$ and $3.4 \mathrm{~m}$ at the three inner-shelf sites (buoy \#1,\#2,\#3 shown in Figure 1) by using the annual maxima from 1979 to 2013 in this study. Shown by Li et al. (2018) for the case with the same windsource (ERA-interim from 1979-2013) and similar GEV models, the estimates of $H_{100}$ were $2.5 \mathrm{~m}$ at buoy \#1, $2.2 \mathrm{~m}$ at buoy\#2, and $3.8 \mathrm{~m}$ at buoy \#3, respectively. When Li et al.,(2018) included a parametric typhoon model in the wind forcing, the estimates of $H_{100}$ by their study increased only by up to $0.15 \mathrm{~m}$. Comparisons to Shi et al., (2019) for the estimates of $H_{100}$ based upon a longer sampling length (39 years from 1979 to 2013) revealed that the bias were limited to $-0.3 \mathrm{~m}$ for buoy \#1 and bouy \#2 and a bit larger $(-0.6 \mathrm{~m})$ for bouy \#3. The negative deviation from their estimates could come from several sources, such as the wind data, bathymetric accuracy, and statistic models. Besides the differences introduced by the statistical models, we deemed that bathymetric accuracy could introduce unavoidable biases between ours and the latest studies. In both Li et al., (2018) and Shi et al., 2019), the bathymetric data interpolated from the General Bathy-metric Chart of the Oceans (GEBCO) was used. The bathymetry of GEBCO failed to represent the submarine profiles of the JRSR, which could highly possible to lead an overestimation on the $H_{100}$. For instance, in the absence of the northern shoals, the buoy \#3 would experience frequent instruction of the northerly swells. In earlier works by Chen et al. (2013) and Li et al. (2016), the extreme waves on the inner shelf of SYS were not well resolved, which possibly because the focused spatial scale was much larger. Ignorance of the JRSR was apparent from their $H_{100}$ maps as the contour lines were normal to the shore, whereas in the reality the SWH were significantly reduced due to the presence of the JRSR.

Due to the unavailability of the long-term observational data for waves in the Yellow Sea, a lookup table dated back to 1990s (Yao et al., 1992) for observational verification. The lookup table was made from the annual maxima of SWH collected from 1960-1989 in the coastal stations of South Yellow Sea. Two numerical datasets of this study from 1979 to 1989 and 1979-1999 were used for calculating the return levels $\left(H_{10}, H_{20}, H_{50}\right.$, and $\left.H_{100}\right)$ for comparisons. The first covered a time period that was included in Yao's dataset. The second had the same sampling length (30-year) as Yao's dataset and contained the earliest samples in this study. The estimates by using 
the annual maxima spanning from 1979-1989 were the closest to Yao's lookup. The biases were within $0.2 \mathrm{~m}$. Whereas by including annual maxima samples after year 1989, the estimates of this study went lower than Yao's estimate. The negative bias could also due to an interestingly negative tendency in the extreme wave climate of the SYS. A further discussion on the long-term tendency of the return levels of extreme SWH were given in the next section.

\subsection{Long-term variability in return level of extreme SWH}

From Figure 10 to Figure 12, there was a decreasing tendency in the return level of extreme SWH, which manifested for the Type I method. A further exploration on the spatial averaged the return levels of extreme SWH revealed such a negative trend was pronounced in the shallow water regime while gradually weakened to the deep water. For example, in the shallow waters with $h<10 \mathrm{~m}$ (shown in Figure 13a), the descending rate of the 100-year return level of SWH versus the increasing sampling length was $0.7 \mathrm{~cm} /$ year with $95 \%$ confidence interval in range of $(-1.0 \mathrm{~cm} / \mathrm{year},-0.4 \mathrm{~cm} / \mathrm{year})$ on a spatial average by using Type I method. A linear fitting of 100-year return level of SWH was statistically significant, with a p-value of 9.38e-06 at 5\% significant level and could explain $49.7 \%$ of the variability in the scatter plot (R-square is 0.4975$)$. The confidence interval for the long-term trend in $h<10 \mathrm{~m}$ regime was narrow (within $0.1 \mathrm{~m}$ with 0.05 significant level). Whilst in deep waters where $h>40 \mathrm{~m}$, the long-term tendency turned positive $(0.01 \mathrm{~cm} /$ year) and was not significant (failed to pass the Fstatistic test) on a spatial average. The CI became wider, which was about $0.15-0.2 \mathrm{~m}$ at both ends. The spatial mean of 100-year return level of extreme SWH in the deep water showed a fierce oscillation which was hard for precise linear fitting (Figure 13b). The negative tendency also appeared by using the other two types of sampling methods (refer to Supplemental Information). On a spatial average, the long-term tendency was in the range of $(-0.2 \mathrm{~cm} /$ year, $-0.4 \mathrm{~cm} /$ year $)$ for the 100 -year return level of extreme $\mathrm{SWH}$ by using the Type II method and in the range of $(-0.1 \mathrm{~cm} /$ year, $-0.5 \mathrm{~cm} /$ year $)$ by using the Type III method. In these two methods, the negative tendencies were found all significant whilst in one order smaller magnitude than that found with Type I method.

In order to distinguish whether this negative tendency was due to a temporal variability or just a statistical convergence in response to the reduced statistical errors, a moving window with a fixed sampling length was applied to the 40-year long hindcasted dataset to calculate the return levels of SWH. The window-width was chosen as 26-year with reference to Mendez et al., (2006; 2008). The starting year was set as 1979 and the window moved at 1-year time interval until the window spanned from year 1993 to year 2018. The prototype was set for window started from 1979 and the return levels obtained from samples started in later years were compared to the prototype by using each type of method (Figure 14). It was found the return level of extreme SWH on the SYS was not random, but changed with time. For instance, for regimes with $h<40 \mathrm{~m}$, the trends between the yearly maxima, monthly maxima and POT were almost decreasing at the similar pace. The largest negative bias was about $-9 \%$ by using the Type I method, and $-5 \%$ by using the Type II and Type III. But for $h>40 \mathrm{~m}$, the trends were heterogeneous. For instance, the return levels of SWH decreased firstly and then 
increased by up to $8 \%$ when the data samples were after 2005 by using the Type I method. Similar fluctuation rather than consistent decrease also revealed by using Type II and Type III methods. This means the more offshore, the more fluctuation in the temporal variability of the extreme waves would appear.

The sources of the negative tendency of the return level of extreme SWH were buried in their sampling sources. Therefore, the long term variability of the annual maxima, monthly maxima and POT were examined and represented by the spatial means (Figure 15). Although the spatial mean could wipe out the local characteristics and make the tendency insignificant ( $p>0.05$ ), negative trends (up to $-0.2 \mathrm{~cm} /$ year) appeared for all cases: annual maxima, monthly maxima and POT. Separated by $h=40 \mathrm{~m}$, it was also found the negative tendency was more obvious in shallower waters (dark dashed lines) than in deeper waters (grey dashed lines) for all types of sampling methods. The spatial variability of the long-term trends could not be ignored and it was highly correlated to the genesis and propagation of the extreme waves (e.g. Menendez et al., 2008; Kukulka et al., 2017). In the SYS, the most severe waves occurred in the up north where $h>40 \mathrm{~m}$. These severe waves often come along with either the southward Siberian cold current. A few were associated with the passages of the tropical cycles in the middle of the Yellow Sea. The former could induce strong and prevailing northerly to northeasterly winds, while the latter could generate storm waves. The extreme waves sourced from the above genesis, however, could be dampened or sheltered by the JRSR on their southward route. Thus, for areas with $h<40 \mathrm{~m}$ of the SYS, there appeared lower extremes and less variability.

The negative tendency was rare and against most of the examples observed in the mid latitude of the north hemisphere where positive tendency in extreme waves were mostly reported: e.g. northeastern Pacific Ocean (e.g. Mendez, 2008; Mendez et al., 2006); Northeast Atlantic (e.g. Wang and Swail, 2001). Most the previous studies attribute the positive long-term trend to global warming and thus more intense wind fields. However, the negative trend in the return level of extreme SWH was not firstly reported by this study. Yao et al., (2017) by suing NCEP reanalysis and WWIII hindcasted the wave climate from 2001 to 2011 over the entire Pacific Ocean. The downward trend in the yearly averaged SWH were found in the Yellow Sea and Japanese strait. Similar downward trend in extreme SWH was also observed off the west coast of Canada (Menendez et al., 2008). Moreover, in the North Sea and Gulf of Alaska, the long-term trend in the extreme SWH was also significant (Feng et al., 2014a; 2014b; Menedez et al., 2008; Yao et al.,2017). However, maybe due to the sparseness and less threat to coastal communities, the downward trends of the extreme SWH were not been paid much attention around the globe. Potential reasons for the descending tendency of the extreme SWH could related to very complex factors. First of all, although for most the central area of Pacific Ocean, the wind intense did show increasing tendency, but the increasing tendency of wind was insignificant in the Yellow Sea area (Yao et al., 2017; Zheng et al., 2017). Second, the SWH relies not only on the wind intensity, but also on the wind-duration, wind-direction and fetch of the wind. The Yellow Sea can be regarded as a semi-enclosed embayment on the map of North Pacific Ocean, which was sheltered by the Japanese islands and Korean 
Peninsula, thus avoiding from the intrusion of big swells generated from the Pacific Center. Besides, wind response to either global warming or climate variability such as El Nin o activity is an ocean-atmospheric interaction based on global-scale (Menendez et al., 2008). Thus, it would have less impact on the regional wind field whereby wave field such as Yellow Sea. Lastly, waves in the SYS were in mixed sea-states. Besides offshore swells, local wind with changing direction or decay could all result in swells, which later can transform and became steep under bathymetric effect while avoid direct influence from wind. Spatially inhomogeneous response of tidal range in response to global warming were extensively reported around the globe (Pickering et al., 2017; Feng et al., 2019), whereas the inhomogeneous response in extreme waves' feedback to long-term climate variability were not paid much attention. This study emphasized requirement for further investigation on the spatial variability in the responses of extreme SWHs to climate change.

The above analysis also showed that as the critical level for defining the extreme SWH lowered down, the slope of long-term trend became milder. This could arise from the natural characteristics of the sampling source. Usually, the yearly maxima contained more randomness (Feng et al. 2014a, 2014b). Note the time series of the yearly maxima showed the largest scatter than the other two (Figure 15a), which helped to explain why the variability of by using Type I method was the largest (Figure 14a and Figure 11), except for $H_{10,10}$ from Type III. A sensitivity tests proposed by Feng et al., (2014b) revealed that the SWH with larger percentile of the exceedance probability obtained better correlation with climate variability. For example, 50th percentile of SWH, which meant the mean value of SWH showed better correlation with sea level pressure anomalies compared to the 2nd percentile of SWH, which indicated an extreme SWH. In this study, the monthly maxima and POT showed more consistent long-term tendency of extreme SWH, possibly because they included more extreme SWH samples.

\section{Conclusions}

The present work conducted a comprehensive sensitivity analysis on generating return value of SWH from the engineering-oriented perspectives. The SYS was chosen as the study area, and 40 years (1979-2018) of wave height hindcasts using SWAN wave model forced with ERA-Interim winds were analyzed. We compared three types of EVA methods for varying return levels of significant wave height (SWH), including the sensitivity of those methods to water depth and sampling length. Type I and Type II methods used the GEV model for best fitting extreme SWH values, but they were differentiated with extreme sampling method: annual maxima and monthly maxima, respectively. Type III method was based on GPD model with modified POT sampling method, in which localized SWH thresholds were determined in a range of $1.3-2.6 \mathrm{~m}$ in a pre-processed analysis.

The reliability of statistic models on wave height estimates vibrates as uncertainties grow with various aspects. As such, this study provides a useful insight into the selection of statistical methods. This study considers a balance between the 
engineering designing standard, data availability, and location for return level estimates. The major findings are as follows.

First, the optimal EVA method which has the most conservative estimations of return period SWH was suggested at each grid point for $H_{100}$ estimation on the shelf of SYS. The Type III method based on a spatially-and-temporally varied POT sampling method was found most conservative and confident for estimating extreme SWH with larger return period $(T)$.

Second, a graphic lookup table of return levels of extreme SWH at 0.05 significant level with reference to the sampling length, return level, and water regimes was provided for readers to make choices in applications. Limitations of each method was easily to be detected (Figure 11 and Supplemental Information). For instance, as the return level decreased (e.g. $H_{100}$ vs. $H_{10}$ ), the confidence interval narrowed for the Type I and Type II method, while widened for the Type III method. Thus, the spatially-and-temporally varied POT sampling method should be carefully applied when estimating extreme SWH with a short $T$. The Type II method based on monthly maxima were the least sensitive to the sampling length, but it would underestimate the return level of extreme SWH, particularly for a large $T$.

Third, this study shows that the spatial variability of return levels of extreme SWH within a regime (e.g. $h \leq 10 \mathrm{~m}, 10 \mathrm{~m}<h \leq 20 \mathrm{~m} 20 \mathrm{~m}<h \leq 40 \mathrm{~m}, h>40 \mathrm{~m}$ ) were not sensitive to sampling length nor to the different types of statistical method. The deviation in the extreme SWH due to the spatial variability were vulnerable to water depth and increased in regimes close to shore. This implies for wave buoy deployment, it is more economical to set less measuring site for wave observations in the open sea, while arrange more near shore.

Nevertheless, the uncertainties aroused from the statistical model always reduced as the sampling length increased, thus long-time dataset were always welcomed for improving the reliability of the return level estimation. Limited by the sparseness of the long-term observational data, the existing studies of extreme SWH were rich in hind-casted numerical datasets, but limited for in situ datasets. Verification on the estimation of return level of extreme SWH often failed through observations, including this piece of work. Thus establishment of an open and well-maintained platform for the long-term wave observation were in urgent demanding for scientific research and engineering application.

Last, the estimation of return levels of extreme SWH from this study were comparable in magnitude to the existing ones based on older data source, but a negative bias apart from the errors induced by different model setup occurred. The negative biases after examination were found relevant to the temporal variability in the nature of extreme SWH samples. Further exploration revealed a long-term negative tendency in the annual maxima, monthly maxima and POT on the inner shelf of the SYS. The negative tendency in the extreme SWH enhanced from deep waters to shallow waters. Future investigation on the contributors to the descending trend of the extreme SWH would be necessary. Time-dependent statistical models might be in-need in the 
following studies to unveil the return levels' co-oscillations with certain climate indices. 


\section{Acknowledgement}

We would like to give our acknowledgement to National Natural Science Foundation of China [No. 51709091]; Natural Science Foundation of Jiangsu Province [No. BK20170874]; and the Fundamental Research Funds for the Central Universities [No. 2017B00514] for the funding support.

\section{REFERENCES}

Appendini, C. M., Torres-Freyermuth, A., Salles, P., López-González, J., \& Mendoza, E. T. (2014). Wave climate and trends for the Gulf of Mexico: A 30-yr wave hindcast. Journal of Climate, 27(4), 1619-1632.

Arkhipkin, V. S., Gippius, F. N., Koltermann, K. P., \& Surkova, G. V. (2014). Wind waves in the Black Sea: results of a hindcast study. Natural Hazards and Earth System Sciences, 14(11), 2883.

Booij, N., Ris, R. C., Holthuijsen, L. H. (1999). A third-generation wave model for coastal regions: 1. model description and validation. J. Geophys. Res: Oceans, 104(C4), 7649-7666.

Caires, S., \& Sterl, A. (2005). 100-year return value estimates for ocean wind speed and significant wave height from the ERA-40 data. Journal of Climate, 18(7), 1032-1048.

Cavaleri, L., \& Sclavo, M. (2006). The calibration of wind and wave model data in the Mediterranean Sea. Coastal Engineering, 53(7), 613-627.

Chen, H., F. Hua, and Yeli Yuan. (2006). "Seasonal characteristics and temporal variations of ocean wave in the Chinese offshore waters and adjacent sea areas." Advances in Marine Science 24.4: 407.

Chen, Y., Xie, D., Zhang, C., \& Qian, X. (2013). Estimation of long-term wave statistics in the East China Sea. Journal of Coastal Research, 65(sp1), 177-182.

Zheng, Chongwei, Zhang Ren, Shi Weilai, Li Xin, and Chen Xuan. (2017) Trends in significant wave height and surface wind speed in the China Seas between 1988 and 2011. J. Ocean Univ. China, 16, 717-726 (2017).

Yao, Xu, Bi Fan, Jinbao Song, Hailun He (2017) The temporal and spatial variations in the Pacific wind and wave fields for the period 2002-2011. Acta Oceanol. Sin., 2017, 36(3), 26-36.

Coles, S., Bawa, J., Trenner, L., \& Dorazio, P. (2001). An introduction to statistical modeling of extreme values (Vol. 208, p. 208). London: Springer.

Cox, A. T., \& Swail, V. R. (2001). A global wave hindcast over the period 1958-1997: Validation and climate assessment. Journal of Geophysical Research: Oceans, 106(C2), 2313-2329.

Federal Emergency Management Agency (FEMA). 2005. Final Draft Guidelines for Coastal Flood Hazard Analysis and Mapping for the Pacific Coast of the United 
States. FEMA Study Contractor: Northwest Hydraulic Consultants, inc. (http://www.fema.gov/plan/prevent/fhm/)

Feng, S., \& Hu, Q. (2008). How the North Atlantic Multidecadal Oscillation may have influenced the Indian summer monsoon during the past two millennia. Geophysical Research Letters, 35(1).

Feng, W., Zhang, Y., Peng, X., Zhang, S., Feng, X. (2009). Statistical study of wind waves for china south yellow sea. Proceedings of the 5th International Conference on APAC 2009, Singapore, 13-16 October 2009.

Feng, X., Feng, H., Li, H., Zhang, F., Feng, W., Zhang, W., \& Yuan, J. (2019). Tidal responses to future sea level trends on the Yellow Sea shelf. Journal of Geophysical Research: Oceans, 124(11),7285-7306

Feng, X., and M. N. Tsimplis (2014), Sea level extremes at the coasts of China, Journal of Geophysical Research: Ocean Science, 119, 1593-1608.

Feng, X., Tsimplis, M., Yelland, M., \& Quartly, G. D. (2014a). Changes in significant and maximum wave heights in the Norwegian Sea. Global and Planetary Change,113, 68-76.

Feng, X., Tsimplis, M., Quarty, G., \& Yelland, M. (2014b). Wave height analysis from 10 years of observations in the Norwegian Sea. Continental Shelf Research, 72(2014), 47-56.

Feng, X. Xu, X., (2018). Characteristics of Storm-Induced Odd-Tide in the South Yellow Sea Radial Sand Ridges. Proceedings of the 6th International Conference on Estuaries and Coasts (ICEC-2018), August 20-23, 2018, Caen, France.

Feng, X., Yi, F., Cao, H. J., \& Yang, B. (2018). An observational study on wave characteristics at the Jiangsu Radial Sand Ridges in the South Yellow Sea of China. The Ocean Engineering, 36(1), 62-73.

Feng, X., Zheng, J., \& Yan, Y. (2012). Wave spectra assimilation in typhoon wave modeling for the East China Sea. Coastal Engineering, 69, 29-41.

Goda, Y. (1992). Uncertainty of design parameters from viewpoint of extreme statistics.

Huang, Y., Yin, B., Perrie, W., \& Hou, Y. (2008). Evaluation of GEV model for frequency analysis of annual maximum water levels in the coast of United States. Ocean Engineering, 35, 1132- 1147

Kaas, E., Li, T. S., \& Schmith, T. (1996). Statistical hindcast of wind climatology in the North Atlantic and northwestern European region. Climate Research, 7(2), 97-110.

Kalra, R., Deo, M. C., Kumar, R., \& Agarwal, V. K. (2005). Artificial neural network to translate offshore satellite wave data to coastal locations. Ocean Engineering, 32(16), 1917-1932. 
Kutupoğlu, V., Çakmak, R. E., Akpınar, A., van Vledder, G. P. (2018). Setup and evaluation of a SWAN wind wave model for the Sea of Marmara. Ocean Engineering, 165, 450-464.

Kukulka, T., Jenkins, R. L., Kirby, J. T.,Shi, F., \& Scarborough, R. W. (2017).Surface wave dynamics in Delaware Bay and its adjacent coastal shelf. Journal of Geophysical Research:Oceans, 122, 8683-8706. https://doi.org/10.1002/2017JC013370

Li, J., Chen, Y., Pan, S., Pan, Y., Fang, J., \& Sowa, D. M. (2016). Estimation of mean and extreme waves in the East China Seas. Applied Ocean Research, 56, 35-47.

Li, J., Pan, S., Chen, Y., Fan, Y. M., \& Pan, Y. (2018). Numerical estimation of extreme waves and surges over the northwest Pacific Ocean. Ocean Engineering, 153, 225-241.

Liang, B., Fan, F., Liu, F., Gao, S., Zuo, H. (2014). 22-Year wave energy hindcast for the China East Adjacent Seas. Renewable Energy, 71, 200-207.

Liu, J. F., \& Sun, L. Y. (2000). Characteristic analysis of wind field and sea wave field in the western part of the N. Pacific Ocean. Marine Forecast, 17(3), 54-62.

Lv, X., Yuan, D., Ma, X., Tao, J. (2014). Wave characteristics analysis in Bohai Sea based on ECMWF wind field. Ocean Engineering, 91, 159-171.

Mathiesen, M., Goda, Y., Hawkes, P. J., Mansard, E., Martín, M. J., Peltier, E., ... \& Van Vledder, G. (1994). Recommended practice for extreme wave analysis. Journal of hydraulic Research, 32(6), 803-814.

Martins, E. S., \& Stedinger, J. R. (2001). Generalized maximum likelihood ParetoPoisson estimators for partial duration series. Water Resources Research, 37(10), 2551-2557.

McNeil, A. J. (1997). Estimating the tails of loss severity distributions using extreme value theory. ASTIN Bulletin: The Journal of the IAA, 27(1), 117-137.

Méndez F.J., MenéndezM.,Luceño A., Losada I.J. (2006). Estimation of the long-term variability of extreme significant wave height using a time-dependent POT model. Journal of Geophysics Research, 111 (2006), C07024.

Menéndez, M., Méndez, F. J., Losada, I. J., \& Graham, N. E. (2008). Variability of extreme wave heights in the northeast Pacific Ocean based on buoy measurements. Geophysical Research Letters, 35(22).

Menéndez, M., Mendez, F. J., Izaguirre, C. , Luceno, A. , \& Losada, I. J. . (2009). The influence of seasonality on estimating return values of significant wave height. Coastal Engineering, 56(3), 211-219.

Mentaschi, L., Besio, G., Cassola, F., \& Mazzino, A. (2015). Performance evaluation of Wavewatch III in the Mediterranean Sea. Ocean Modelling, 90, 82-94. 
Moeini, M. H., Etemad-Shahidi, A., \& Chegini, V. (2010). Wave modeling and extreme value analysis off the northern coast of the Persian Gulf. Applied Ocean Research, 32(2), 209-218.

Muir, L. R., \& El-Shaarawi, A. H. (1986). On the calculation of extreme wave heights: a review. Ocean Engineering, 13(1), 93-118.

Muraleedharan, G., Lucas, C., Soares, C. G., Nair, N. U., \& Kurup, P. G. (2012). Modelling significant wave height distributions with quantile functions for estimation of extreme wave heights. Ocean Engineering, 54, 119-131.

Nayak, S., Bhaskaran, P. K., Venkatesan, R. (2012). Near-shore wave induced setup along Kalpakkam coast during an extreme cyclone event in the Bay of Bengal. Ocean Engineering, 55, 52-61.

Niroomandi, A., Ma, G., Ye, X., Lou, S., \& Xue, P. (2018). Extreme value analysis of wave climate in Chesapeake Bay. Ocean Engineering, 159, 22-36.

Niu, Xiaojing and Chen, Yixiang, 2020. Energy accumulation during the growth of forced wave induced by a moving atmospheric pressure disturbance. Coastal Engineering Journal, 62(1),23-34.

Reistad, M., Breivik, Ø., Haakenstad, H., Aarnes, O. J., Furevik, B. R., \& Bidlot, J. R. (2011). A high-resolution hindcast of wind and waves for the North Sea, the Norwegian Sea, and the Barents Sea. Journal of Geophysical Research: Oceans, 116(C5).

Pickering, M. D., Horsburghb, K. J., Blundella, J. R., Hirschic, J. J. M., Nichollsd, R. J., Verlaane, M., \& Wells, N. C. (2017). The impact of future sea-level rise on the global tides. Continental Shelf Research, 142, 50-68. https://doi.org/10.1016/j.csr.2017.02.004

Qi, Y. (1997). A preliminary study on characteristics of wind and wave over the central area of Yellow Sea. Marine Science Bulletin-TianJin, 16, 1-5.

Shi, J., Zheng, J., Zhang, C., Joly, A., Zhang, W., Xu, P., ... \& Chen, T. (2019). A 39year high resolution wave hindcast for the Chinese coast: Model validation and wave climate analysis. Ocean Engineering, 183, 224-235.

Signell, R. P., Carniel, S., Cavaleri, L., Chiggiato, J., Doyle, J. D., Pullen, J., \& Sclavo, M. (2005). Assessment of wind quality for oceanographic modelling in semienclosed basins. Journal of Marine Systems, 53(1-4), 217-233.

Soares, C. G., Weisse, R., Carretero, J. C., \& Alvarez, E. (2002, January). A 40 year hindcast of wind, sea level and waves in European waters. In ASME 2002 21st International Conference on Offshore Mechanics and Arctic Engineering (pp. 669-675). American Society of Mechanical Engineers.

Sterl, A., Komen, G. K., \& Cotton, P. D. (1998). Fifteen years of global wave hindcasts using winds from the European Centre for Medium-Range Weather Forecasts 
reanalysis: Validating the reanalyzed winds and assessing the wave climate. Journal of Geophysical Research: Oceans, 103(C3), 5477-5492.

Wang, X. L., \& Swail, V. R. (2001). Changes of extreme wave heights in Northern Hemisphere oceans and related atmospheric circulation regimes. Journal of Climate, 14(10), 2204-2221.

Wang, X. L., \& Swail, V. R. (2002). Trends of Atlantic wave extremes as simulated in a 40-yr wave hindcast using kinematically reanalyzed wind fields. Journal of climate, 15(9), 1020-1035.

Wang, Y., \& Yi, H. (1997). Statistics charicteristics and spectrum analysis of waves in East China Sea. Journal of Dalian Fisheries University, 12(3), 33-39.

Wang, Z. F., Wu, K. J., Zhou, L. M., \& Wu, L. Y. (2012). Wave characteristics and extreme parameters in the Bohai Sea. China Ocean Engineering, 26(2), 341-350.

Xu, S., Huang, W. (2011). Estimating extreme water levels with long-term data by GEV distribution at Wusong station near Shanghai city in Yangze Estuary. Ocean Engineering, 38, 468-478.

Yao, G., Ma, Z., Ding, B., Wang, J. (1992). Statistical Analysis of winds and waves in the Yellow Ses and the Bohai Sea. China Harbour Engineering, 1992(04), 3137.

Yang, B., Feng, W. B., \& Zhang, Y. (2014). Wave characteristics at the south part of the radial sand ridges of the southern Yellow Sea. China Ocean Engineering, 28(3), 317-330.

Zhao, J. (2020). Characteristics and Influencing Factors of Wind-seas and Swells in Radial Sand Ridges: 19-25 (Master's thesis). Nanjing, Jiangsu: Hohai University. 